www.jmscr.igmpublication.org

Impact Factor 5.84

Index Copernicus Value: 83.27

ISSN (e)-2347-176x ISSN (p) 2455-0450

crossref DOI: https://dx.doi.org/10.18535/jmscr/v5i2.71

Journal Of Medical Science And Clinical Research

\title{
Oral Mucosal Changes in Tobacco Users- A Cytomorphometric Analysis
}

\author{
Authors \\ N. Mohan ${ }^{1}$, Anju Mathew ${ }^{2}$, Sabitha Gokulraj ${ }^{3}$ \\ ${ }^{1,2,3}$ Dept of Oral Medicine and Radiology, Vinayaka Mission Sankarachariyar Dental College, Salem, India \\ Corresponding Author
}

Anju Mathew

Email: dranjumathew@gmail.com, 9566629596

\begin{abstract}
Aim: To assess the effect of tobacco smoking and tobacco chewing on the buccal mucosa by cytomorphometry. Materials and Methodology: Oral mucosal cells were collected from 45 subjects divided into 3 groups: Tobacco Smokers, Tobacco Chewers and Controls. The cells were stained using Papanicolaou stain and viewed under high power microscope. Cytomorphometric analyses of the cells in captured image were analysed using Image $J$ analysis software to find out the Cellular Diameter, Nuclear Diameter and Nuclear Cytoplasmic Ratio. The statistical test used was ANOVA and $p<0.05$ was considered significant.

Results: The cellular diameter gradually decreases while nuclear diameter and nuclear cytoplasmic ratio increases from controls to smokers and to chewers.

Conclusion: The results confirmed that tobacco using influenced the cytomorphology of normal buccal mucosa and the degree of these changes was found to be greater in chewers compared to smokers. Cytomorphometric changes can be an early indicator of cellular alterations.

Keywords: Cytomorphometry, Exfoliative Cytology, Cellular diameter, Nuclear Diameter, Nuclear Cytoplasmic Ratio
\end{abstract}

\section{Introduction}

Cancer is derived from a Greek word 'Karkinos' which means crab, representing how carcinoma extends its claws like a crab into adjacent tissues. ${ }^{[1]}$ Cancer is the second most common cause of death after heart disease. ${ }^{[2]}$ Oral cancer ranks $11^{\text {th }}$ most cancer in the world. ${ }^{[3]}$ It is the $12^{\text {th }}$ most common cancer in women and $6^{\text {th }}$ in men. ${ }^{[2]}$ India accounts for one - third of the world's oral cancer resulting from unhealthy hygienic practices and oral habits including consumption of smoked or smokeless tobacco. ${ }^{[3]}$

The strong association between oral cancers with the use of tobacco is well established. All of the major forms of tobacco use are known to cause oral cancer. The magnitude of the risks increases with greater amounts or longer duration of tobacco usage. Tobacco contains carcinogens that influence the DNA repair, cell cycle control and may produce chromosomal aberrations. Most oral cancer lesions are preceded by premalignant changes in oral mucosa, which could be of great benefit in early diagnosis of these lesions. ${ }^{[4]}$

Exfoliative cytology is a simple, non- invasive, less expensive diagnostic technique which increases the chances of timely detection of potentially malignant and malignant disorders. ${ }^{[5]}$ Cytomorphometry is a computer-assisted analysis of images formed by a microscope which increases the sensitivity of 
exfoliative cytology for early diagnosis of potentially malignant lesions, since this technique is precise, objective, and reproducible. Various quantitative parameters such as nuclear size, cell size, nuclear - to - cytoplasmic ratio, nuclear shape, and nuclear discontinuity can be evaluated to confirm the diagnosis. Out of these parameters, nuclear size, cellular size and their ratio shows greater significance in evaluating oral lesions. ${ }^{[6]}$

Taking this into reflection the present study has been carried out to assess the effect of tobacco chewing and smoking on buccal mucosa and compare the cytomorphology of cells collected from buccal mucosa of tobacco chewers and smokers with those of tobacco non-users.

\section{Material and Methodology}

The sample for this study included patients visiting the Department of Oral Medicine and Radiology. Ethical Clearance was obtained from the Institutional Committee. The sample group comprises of 45 patients divided into 3 subgroups entailing of 15 patients in each subgroup.

Group I - Tobacco Chewers

Group II - Tobacco Smokers

Group III - Controls.

Patients of age group $20-60$ years, with tobacco chewing and smoking habits for more than 3 times a day for more than 5 years were included for the study. Patients without any tobacco using habits were included in control group. Patients with systemic diseases like anaemia and Diabetes Mellitus, patients who had undergone radiotherapy or chemotherapy, habit of alcoholism, under long term medication and with clinical evident lesions in the oral cavity were excluded from the study.

All the patients selected for the study were explained in detail about the need for the study. Individually voluntary informed consent was obtained from the patients. Clinical examination was performed and data was recorded in a proforma.

The area to be smeared was first wiped clean of excessive saliva and surface debris. Scraping of surface cells from the selected area was obtained with a moistened wooden spatula and the material obtained was immediately spread on a fresh, clean and dry slide. The slides were fixed with alcohol and stained using the Rapid Pap kit, following the manufacturer's instructions.

The kit consists of Rapid PAP nuclear stain, hematoxylin stain, cytoplasmic stain, combination of OG-6 and Light Green-eosin, Scott's tap water buffer, and dehydrant solution, and propanol and xylene. Smears were fixed in tap water followed by Rapid PAP nuclear stain for $45 \mathrm{~s}$. The smears were washed in Scott's tap water for $30 \mathrm{~s}$ followed by Rapid PAP dehydrant for $60 \mathrm{~s}$ and cytoplasmic stain for $45 \mathrm{~s}$. Then it was washed in Scott's tap water followed by dehydrant for $30 \mathrm{~s}$. The slide was airdried and then dipped in xylene and mount wet. ${ }^{[7]}$

The stained smears were examined under a high power microscope as shown in Figure 1. The images were transferred to a computer via a camera (Sony W830, India) and analysed using a software ImageJ analyser (1.41o/ Java 1.8.0_91). Only clearly defined cells were measured, avoiding clumped or folded cells, and unusually distorted nuclei and cells. The sampling was done in a stepwise manner, moving the slide from the left upper corner to the right, and then down in order to avoid measuring the same cells twice. Image analysis was performed to obtain mean cellular diameter, nuclear diameter and nuclear cytoplasmic ratio.

Measurements were obtained from 100 cells in both axes for the cell and for the nucleus. The average of the values from both axes was considered as the diameter of the cell and the nucleus and was recorded as shown in Figure 2. The data was analysed by a second observer and mean values were obtained. The mean values of cell diameter and nuclear diameter of all 100 cells were calculated and recorded.

\section{Statistical Analysis}

Analysis of variance (one-way ANOVA) was performed for the three groups to compare the mean of cellular diameter, nuclear diameter and ratio of nuclear diameter to cellular diameter. $\mathrm{P}<0.05$ was considered statistically significant. 


\section{Results}

This study was conducted on 45 individuals that included 15 tobacco chewers, 15 tobacco smokers with habit of chewing and smoking more than 3 times a day for more than 5 years and 15 controls without any tobacco using habits. The mean cellular diameter, mean nuclear diameter and mean nuclear cytoplasmic ratio was analysed as shown in Figure 3 and Figure 4. There was significant difference between the three groups. The mean cellular diameters decreased while the mean nuclear diameters and mean nuclear cytoplasmic ratios increased from controls to tobacco smokers to tobacco chewers. The results were also analysed with respect to duration, age group and type of tobacco used as shown in Figure 5, Figure6 and Figure 7. There was significant difference present within age group and type of tobacco used. There was no significant difference between the groups with respect to duration.

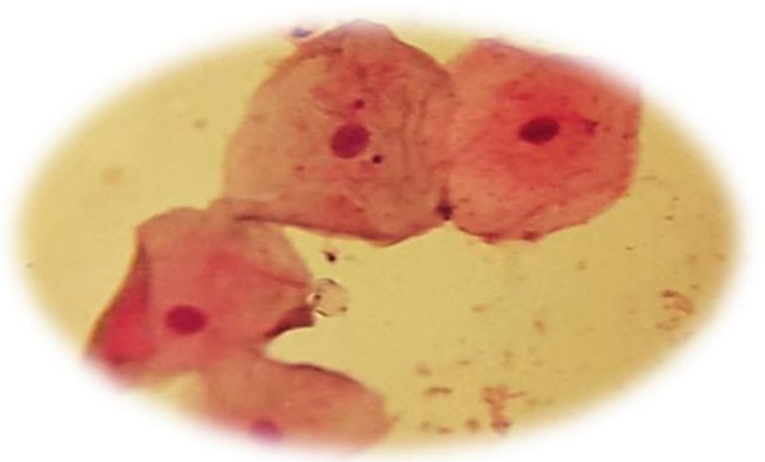

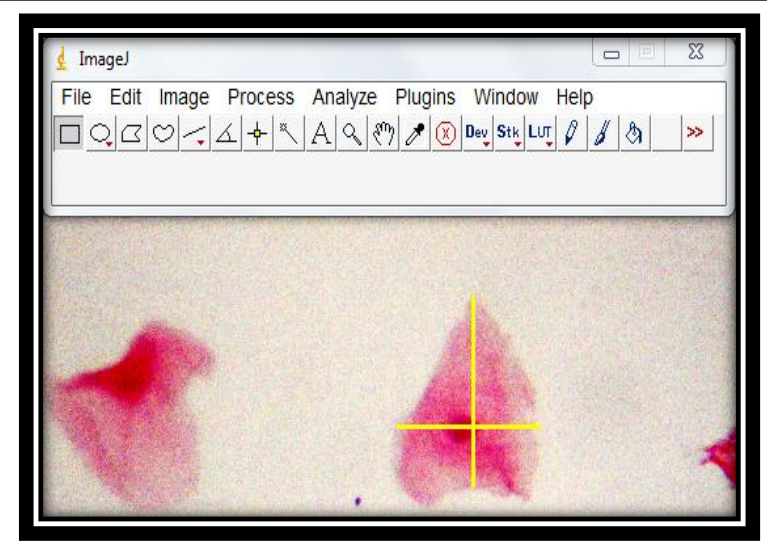

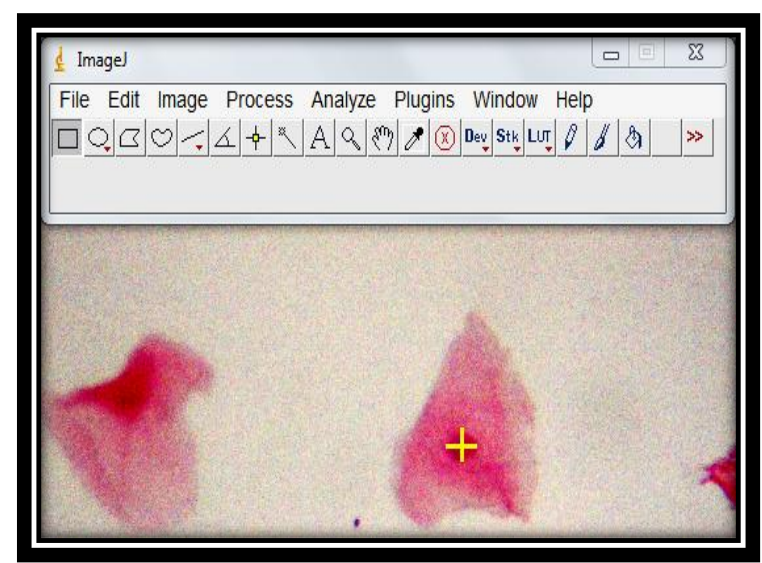

Figure 2: Measurements done using software

Figure 1: Cells viewed under high power microscope

Figure 3: Comparison between the Subgroups

\begin{tabular}{|c|c|c|c|c|c|c|}
\hline & & $\mathrm{N}$ & Mean & SD & ANOVA & $\mathrm{p}$ \\
\hline \multirow{3}{*}{ Cellular Diameter } & Smokers & 15 & 66.00 & 2.64 & \multirow{3}{*}{352.82} & \multirow{3}{*}{$<0.001 * *$} \\
\hline & Chewers & 15 & 58.16 & 2.02 & & \\
\hline & Control & 15 & 78.54 & 1.56 & & \\
\hline \multirow{3}{*}{ Nuclear Diameter } & Smokers & 15 & 8.54 & 0.34 & \multirow{3}{*}{104.49} & \multirow{3}{*}{$<0.001^{*} *$} \\
\hline & Chewers & 15 & 9.74 & 0.58 & & \\
\hline & Control & 15 & 7.42 & 0.35 & & \\
\hline \multirow{3}{*}{ Nuclear cytoplasmic Ratio } & Smokers & 15 & 0.12 & 0.01 & \multirow{3}{*}{198.55} & \multirow{3}{*}{$<0.001 * *$} \\
\hline & Chewers & 15 & 0.16 & 0.01 & & \\
\hline & Control & 15 & 0.09 & 0.01 & & \\
\hline
\end{tabular}

** Significant at $1 \%$

Since the $\mathrm{p}$ value is less than 0.05 there is a significant difference in mean cellular diameter, Nuclear Diameter and Nuclear cytoplasmic Ratio was found between the groups. 


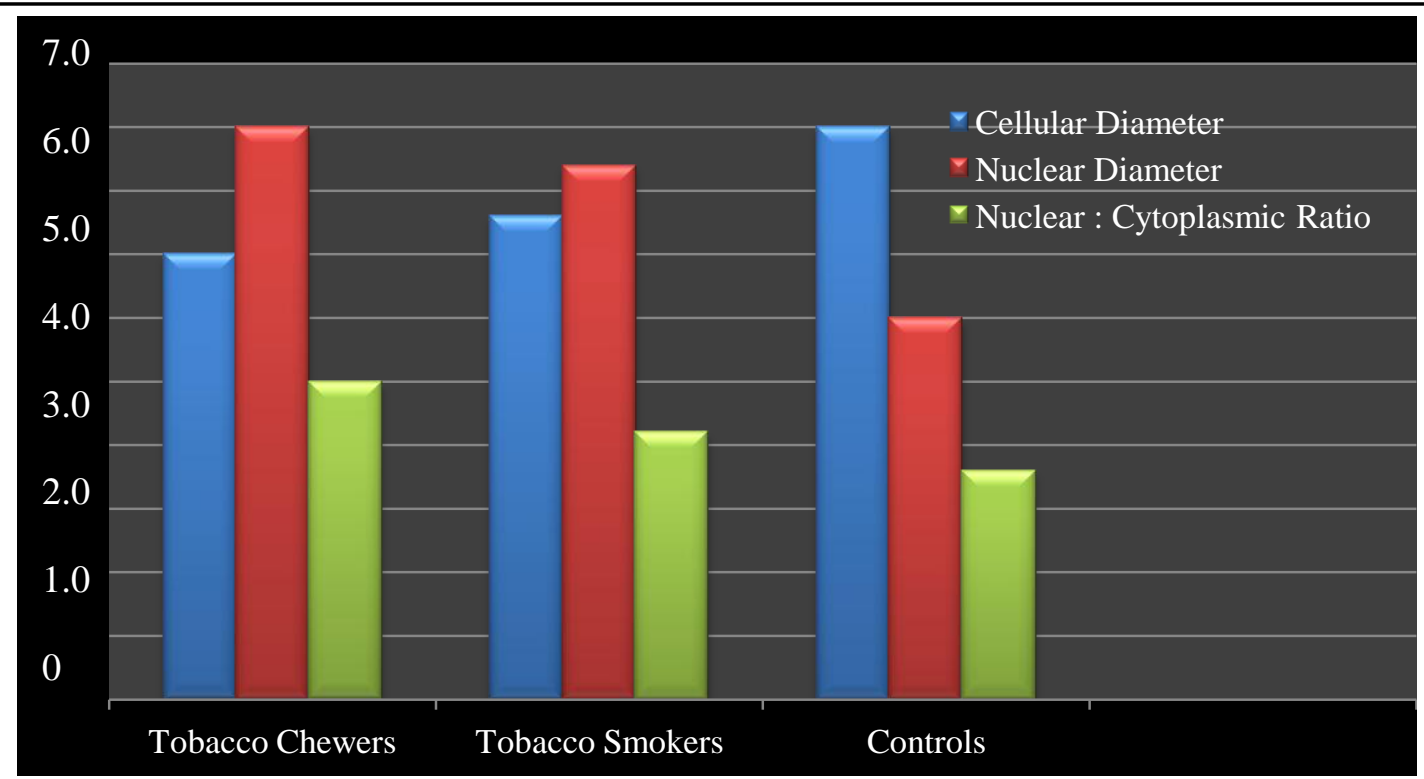

Figure 4 demonstrating cellular diameter decreases while the nuclear diameter and nuclear cytoplasmic ratio increases from controls to smokers to chewers

Figure 5: Comparison in Respect to Duration

\begin{tabular}{|c|c|c|c|c|c|c|}
\hline & Duration & $\mathrm{N}$ & Mean & SD & ANOVA & $\mathrm{p}$ \\
\hline \multirow{4}{*}{ Cellular Diameter } & $5-10$ & 14 & 60.74 & 5.04 & \multirow{4}{*}{1.22} & \multirow{4}{*}{0.310} \\
\hline & $11-15$ & 6 & 63.93 & 4.17 & & \\
\hline & $16 \&$ above & 10 & 62.83 & 4.05 & & \\
\hline & Total & 30 & 62.08 & 4.61 & & \\
\hline \multirow{4}{*}{ Nuclear Diameter } & $5-10$ & 14 & 9.34 & 0.89 & \multirow{4}{*}{2.36} & \multirow{4}{*}{0.114} \\
\hline & $11-15$ & 6 & 8.57 & 0.46 & & \\
\hline & $16 \&$ above & 10 & 9.20 & 0.59 & & \\
\hline & Total & 30 & 9.14 & 0.77 & & \\
\hline \multirow{4}{*}{ Nuclear cytoplasmic Ratio } & $5-10$ & 14 & 0.15 & 0.02 & \multirow{4}{*}{2.09} & \multirow{4}{*}{0.143} \\
\hline & $11-15$ & 6 & 0.13 & 0.02 & & \\
\hline & $16 \&$ above & 10 & 0.14 & 0.02 & & \\
\hline & Total & 30 & 0.14 & 0.02 & & \\
\hline
\end{tabular}

Since the $\mathrm{p}$ value is greater than 0.05 there is no significant difference in mean cellular diameter, Nuclear Diameter and Nuclear Cytoplasmic Ratio was found with respect to duration.

Figure 6: Comparison in Respect to Age Group

\begin{tabular}{|c|c|c|c|c|c|c|}
\hline & Age & $\mathrm{N}$ & Mean & SD & $\mathrm{t}$ & $\mathrm{p}$ \\
\hline \multirow{2}{*}{ Cellular Diameter } & $20-40$ & 22 & 69.86 & 8.72 & \multirow{2}{*}{1.76} & \multirow{2}{*}{0.085} \\
\hline & $41-60$ & 23 & 65.37 & 8.36 & & \\
\hline \multirow{2}{*}{ Nuclear Diameter } & $20-40$ & 22 & 8.21 & 1.06 & \multirow{2}{*}{2.33} & \multirow{2}{*}{$0.025^{*}$} \\
\hline & $41-60$ & 23 & 8.91 & 0.94 & & \\
\hline \multirow{2}{*}{ Nuclear cytoplasmic Ratio } & $20-40$ & 22 & 0.12 & 0.03 & \multirow{2}{*}{1.87} & \multirow{2}{*}{0.068} \\
\hline & $41-60$ & 23 & 0.13 & 0.03 & & \\
\hline
\end{tabular}

*Significant at $5 \%$

Since the $\mathrm{p}$ value is less than 0.05 there is a significant difference in mean Nuclear Diameter was found between the age groups. 
Figure 7: Comparison in Respect to Habit

\begin{tabular}{|c|c|c|c|c|c|}
\hline & Habit & Mean & SD & ANOVA & $\mathrm{p}$ \\
\hline \multirow{6}{*}{ Cellular Diameter } & Nil & 78.541 & 1.563 & \multirow{6}{*}{148.62} & \multirow{6}{*}{$<0.001 * *$} \\
\hline & Beedi & 66.765 & 2.806 & & \\
\hline & Cigarette & 65.487 & 2.552 & & \\
\hline & Tobacco & 58.868 & 1.468 & & \\
\hline & $\begin{array}{c}\text { "Betel } \\
\text { nut with Tobacco" }\end{array}$ & 58.675 & 1.500 & & \\
\hline & Hans & 56.485 & 2.710 & & \\
\hline \multirow{6}{*}{ Nuclear Diameter } & Nil & 7.423 & 0.354 & \multirow{6}{*}{47.45} & \multirow{6}{*}{$<0.001 * *$} \\
\hline & Beedi & 8.728 & 0.275 & & \\
\hline & Cigarette & 8.414 & 0.329 & & \\
\hline & Tobacco & 10.070 & 0.579 & & \\
\hline & $\begin{array}{c}\text { "Betel } \\
\text { nut with Tobacco" }\end{array}$ & 9.497 & 0.559 & & \\
\hline & Hans & 9.688 & 0.555 & & \\
\hline \multirow{6}{*}{ Nuclear cytoplasmic Ratio } & $\mathrm{Nil}$ & 0.087 & 0.006 & \multirow{6}{*}{78.27} & \multirow{6}{*}{$<0.001 * *$} \\
\hline & Beedi & 0.120 & 0.009 & & \\
\hline & Cigarette & 0.124 & 0.009 & & \\
\hline & Tobacco & 0.166 & 0.015 & & \\
\hline & $\begin{array}{c}\text { "Betel } \\
\text { nut with Tobacco" }\end{array}$ & 0.158 & 0.015 & & \\
\hline & Hans & 0.163 & 0.015 & & \\
\hline
\end{tabular}

Since the $\mathrm{p}$ value is less than 0.05 there is a significant difference in mean cellular diameter, Nuclear Diameter and Nuclear cytoplasmic Ratio was found with respect to Habit.

\section{Discussion}

Oral cancer is a tobacco related disease which marks for almost $40 \%$ of all cancers in Indian subcontinent. ${ }^{8}$ All of the main forms of tobacco use like cigarettes, cigars, pipes and smokeless tobacco (chewing tobacco and snuff) are prime suspects in causing oral cancer. ${ }^{[7]}$

During transformation of normal tissue to premalignancy or malignancy, cellular variations occur much before they are seen under the microscope and before clinical variations become evident. Identification of high-risk oral premalignant lesions and intervention at premalignant stages could establish one of the solutions in dipping the mortality, morbidity and cost of treatment linked with oral squamous cell carcinoma. ${ }^{[5]}$

The role of exfoliative cytology in the detection of pre-malignant and malignant lesions has created several controversies. Oral exfoliative cytology is a simple, non-invasive, less time consuming procedure with sensitivity of $89 \%$ and specificity of $89.5 \%$ while few researches strongly criticize that there is no role for exfoliative cytology in early cancer detection since it is not $100 \%$ sensitive. Even though exfoliative cytology is not $100 \%$ accurate, it has its peculiar potential value in cases where biopsy is contraindicated like in systemically compromised patients, inaccessible areas, recurrent malignancies and in mass screening. The smear acquiredby exfoliative cytology can be analysed quantitatively and qualitatively. ${ }^{[9]}$

Cytomorphometry is a computer-assisted technique for the analysis of cells. A computer analysis a digital microscopic image of the collected cells with the help of a neural network based image processing system. ${ }^{[7]}$ With advancements in the field of quantitative oral exfoliative cytology various parameters such as cellular diameter, nuclear diameter, nuclear-to-cytoplasmic ratio, nuclear shape, nuclear discontinuity, optical density and nuclear texture can be assessed collectively in order to confirm the diagnosis. Of these parameters, cellular diameter, nuclear diameter and their ratio have been shown to be remarkable in the evaluation of oral lesions. ${ }^{[9]}$ 
Cowpe et al.demonstrated that exfoliative cytology is capable of detecting malignant changes through estimation of ratio of nuclear size to cytoplasmic size, using planimeter method in Papanicolaoustained smears. ${ }^{[10]}$ Remmerbach et al. evaluated the reliability of oral cytology and its cytomorphometric analysis in the early detection of oral cancer. The results of the study showed that the sensitivity of the cytology was $94.6 \%$ and specificity $99.5 \%$ respectively. ${ }^{[11]}$ Megha Singh et al. conducted a study on cytological smears obtained from oral lesions of patients with habit of tobacco smoking and tobacco chewing and concluded that tobacco chewing and smoking influenced the cytomorphology of normal appearing buccal mucosa and the degree of these changes were found to be greater in chewers as compared to smokers. ${ }^{[12]}$ D Parmar et al assessed the effect of tobacco chewing on buccal mucosa by using cytomorpho-metry. The results showed there is progressive increase in nuclear parameters, decrease in cellular parameters and increase in ratio of nuclear to cellular parameters in smears from tobacco chewers, as compared with normal subjects. It was concluded that these adaptive changes in the cellular and nuclear parameters tend to be a progression toward dysplastic or premalignant change. ${ }^{[4]}$ Ramaesh et al. evaluated the effect of smoking cigarettes and chewing betel quid and concluded that the nucleardiameter of the oral mucosa cells in individuals both these habits were significantly greater while the cellular diameter decreased than that of the control group individuals. ${ }^{[13]}$ Einstein and Sivapathasundraham analysed the effect of smoking and betel quid chewing on the oral mucosa,using cytomorphological methods, and concluded an increase in the average value of nuclear diameter, and a decrease in cytoplasmic diameter values of smokers and individuals with both these habits. ${ }^{[14]}$

According to Center for Disease Control, chewing tobacco 7-8 times a day may be equivalent to smoking 30-40 cigarettes/day. Though the nicotine content of chewing tobacco is lower than the smoking form, it is said to have an amplified carcinogenic potential because it remains in contact with the oral mucosa for longer periods of time. ${ }^{[15]}$
In our study we assessed the effect of tobacco chewing and smoking on buccal mucosa by cytomorphometry and we found that the cellular diameter decreased while the nuclear diameter and nuclear cytoplasmic ratio increased from the controls to tobacco smokers to tobacco chewers. All the cellular parameters were assessed with respect to age groups, duration and to type of tobacco used. It was found that as there were more cellular alterations as the age advances. There was no significant alteration with respect to duration. The form of tobacco used was also assessed and it presented usage of Hans and tobacco with least cellular diameter and greatest nuclear diameter and their ratio compared to other forms.

The results of this study highlight the significance of early recognition of cellular alterations even in the absence of visible changes of the mucosal surface.

\section{Conclusion}

Oral cancer disguises itself as an innocent lesion in early stages. Patients are usually unaware of the lesion and report to the clinician at a later stage where invasive treatment is required. Judicious detection of potentially malignant and malignant disorders declines morbidity rate and upsurges patient survival rate. This study reveals that the cytomorphometric changes could be the earliest indicators of cellular alterations for identification of individuals who require early intervention even in the absence of visible changes of mucosal surface. Hence, cytomorphometric analysis of oral smears can be carried out regularly in order to detect alterations in the cellular and nuclear dimensions especially among tobacco users. Furthermore, this method can also aid in motivating individuals to withdraw from tobacco use.

\section{References}

1. Antony George, Sreenivasan BS, Sunil S Et al. Potentially Malignant Disorders of Oral Cavity, Oral and Maxillofacial Pathology. 2011 (2) Issue 1. 95- 100

2. Hamed Mortazavi, Maryam Baharvand, Masoumeh Mehdipour. Oral Potentially 
Malignant Disorders: An Overview of More Than 20 Entities. Joddd. 2014. Vol 8(1). 614

3. AcharyaSiddardh, Anup, Binjoo Et al.The Occurrence Of Potentially Malignant Disorders In Oral Cavity :Asurvey From Bagru - Khurd Of Rural Jaipur, Rajasthan, International Journal Of Biomedicine.2014 Vol 4 (1). 11- 14

4. D Parmar, NilimaSawke, Gopalkrishna Sawke. Cytomorphometric Analysis of Buccal Mucosa Cells in Tobacco Chewers. Astrocyte. Jan-Mar 2015 .Volume 1.Issue 4

5. Alka Harish Hande, Minal S. Chaudhary.Cytomorphometric analysis of buccal mucosa of tobacco chewers. Romanian Journal of Morphology and Embryology 2010, 51(3):527-532

6. Musta, Hayati Murat, Cemal. The cytomorphological analysis of buccal mucosa cells in smokers. Turk J Med Sci 2011; 41 (2): 205-210

7. Nivia M, Sunil SN, Rathy R, Anilkumar TV.Comparative cytomorphometric analysis of oral mucosal cells in normal, tobacco users, oral leukoplakia and oral squamous cell carcinoma. J Cytol 2015;32:253-60.

8. Cha IH, Park JY, Chung WY, Choi MA, Kim HJ, Park KK.Polymorphisms of CYP1A1 and GSTM1 genesand susceptibility to oral cancer.Yonsei Med J 2007;48:233-9.

9. Sumit Babuta, RohinGarg, Khushboo Mogra, NehaDagal.Cytomorphometrical Analysis of Exfoliated Buccal Mucosal Cells: Effect of Smoking. Acta Medica International. Jan - Jun 2014 | Vol 1| Issue1 |

10. Cowpe JG, Longmore RB, Green MW. Quantitative exfoliative cytology of normal oral squames: An age, site and sex-related survey. J R Soc Med 1985;78:995-1004.

11. Remmerbach TW, Weidenbach H, Pomjanski N, Knops K, Mathes S, Hemprich $\mathrm{A}$, et al. Cytologic and DNA-cytometric early diagnosis of oral cancer. Anal Cell Pathol 2001;22:211-21.

12. Singh M, Sircar K, Tandon A, Chowdhry A, Popli DB. The role of tobacco as an etiological agent for oral cancer: Cytomorphometrical analysis of the buccal mucosa in tobacco users. Dent Res J 2014;11:649-55.

13. Ramaesh T, Mendis BR, Ratnatunga N, Thattil RO. The effect of tobacco smoking and of betel chewing with tobacco on the buccal mucosa: a cytomorphometric analysis. J Oral Pathol Med 1999; 28: 385-8.

14. Einstein TBA, Sivapathasundharam B. Cytomorphometric analysis of the buccal mucosa of tobacco users. Ind J Dent Res 2005; 16: 42-6.

15. Fant RV, Henningfield JE, Nelson RA, Pickworth WB.Pharmacokinetics and pharmacodynamics of moist snuff in humans.Tob Control 1999;8:387-92. 